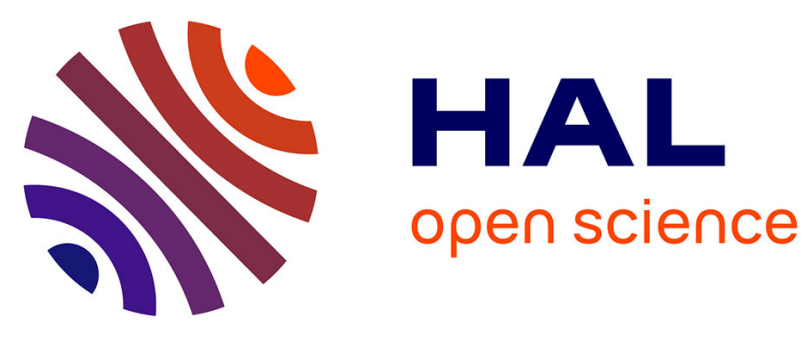

\title{
Hyperfine Interactions and Slow Spin Dynamics in Quasi-isotropic InP-based Core/Shell Colloidal Nanocrystals
}

Annalisa Brodu, Mickaël Tessier, Damien Canneson, Dorian Dupont, Mariana V Ballottin, Peter C M Christianen, Celso de Mello Donega, Zeger Hens, Dmitri R Yakovlev, Manfred Bayer, et al.

\section{To cite this version:}

Annalisa Brodu, Mickaël Tessier, Damien Canneson, Dorian Dupont, Mariana V Ballottin, et al.. Hyperfine Interactions and Slow Spin Dynamics in Quasi-isotropic InP-based Core/Shell Colloidal Nanocrystals. ACS Nano, 2019, 13 (9), pp.10201-10209. 10.1021/acsnano.9b03384 . hal-02394897

\section{HAL Id: hal-02394897 https://hal.science/hal-02394897}

Submitted on 5 Dec 2019

HAL is a multi-disciplinary open access archive for the deposit and dissemination of scientific research documents, whether they are published or not. The documents may come from teaching and research institutions in France or abroad, or from public or private research centers.
L'archive ouverte pluridisciplinaire HAL, est destinée au dépôt et à la diffusion de documents scientifiques de niveau recherche, publiés ou non, émanant des établissements d'enseignement et de recherche français ou étrangers, des laboratoires publics ou privés. 


\title{
Hyperfine Interactions and Slow Spin Dynamics in Quasi-Isotropic InP Core/Shell Colloidal Nanocrystals
}

\author{
Annalisa Brodu,,$^{\S}$ Mickael D. Tessier, "Damien Canneson, ${ }^{*}$ Dorian Dupont, "Mariana V. \\ Ballottin,,$^{\ddagger}$ Peter C. M. Christianen, ${ }^{\ddagger}$ Celso de Mello Donega, ${ }^{\S}$ Zeger Hens,,$"$ Dmitri R. \\ Yakovlev, ${ }^{*}, \star$ Manfred Bayer, ${ }^{*}, \neq$ Daniel Vanmaekelbergh,${ }^{\S}$ and Louis Biadala. ${ }^{\dagger}{ }^{* *}$ \\ ${ }^{\S}$ Debye Institute for Nanomaterials Science, Utrecht University, 3584 CC Utrecht, The Netherlands \\ "Physics and Chemistry of Nanostructures, Ghent University, 9000 Ghent, Belgium \\ ${ }^{*}$ High Field Magnet Laboratory, HFML-EMFL, Radboud University, 6525 ED Nijmegen, The \\ Netherlands \\ ${ }^{*}$ Experimentelle Physik 2, Technische Universität Dortmund, 44227 Dortmund, Germany \\ ${ }^{\ddagger}$ Ioffe Institute, Russian Academy of Sciences, 194021 St Petersburg, Russia \\ ${ }^{\dagger}$ Institut d'Électronique, de Microélectronique et de Nanotechnologie, UMR CNRS 8520, Villeneuve \\ d'Ascq, France
}

*Corresponding author: louis.biadala@isen.iemn.univ-lille1.fr

\section{ABSTRACT}

Colloidal InP core/sell nanocrystals are taking over CdSe-based nanocrystals, notably in optoelectronic applications. Despite their use in commercial device such as display screens, the optical properties of InP nanocrystals and especially their relation with the exciton fine structure remains poorly understood. In this work, we show that the magneto-optical properties of ensemble InP-based core/shell nanocrystals investigated in strong magnetic fields up to $30 \mathrm{~T}$ are strikingly different compared to other colloidal nanostructures. Notably, the mixing of the lowest spin-forbidden dark exciton state with the nearest spin-allowed bright state does not occur, up to the highest magnetic fields applied. This lack of mixing in ensemble of nanocrystals suggests an anisotropy-tolerance of InP nanocrystals. This striking property allowed us to unveil the slow spin dynamics between Zeeman sublevels (up to 400 ns at $15 \mathrm{~T}$ ). Furthermore, we show that the unexpected magnetic field-induced lengthening of the dark exciton lifetime results from the hyperfine interaction between the spin of the electron in the dark exciton with the nuclear magnetic moments. Our results demonstrate the richness of the spin physics in InP quantum dots and stress the large potential of InP nanostructures for spin-based applications.

\section{KEYWORD:}

colloidal nanostructure, III-V, hyperfine interaction, dark exciton, high magnetic field 
Colloidal semiconductor nanocrystals (NCs) with quantum confinement in all three dimensions, also called quantum dots (QDs), remain to attract interest, since they show a bright luminescence that can be spectrally tuned by their size. For the visible spectrum, CdSe NCs have been the work horse, as surface passivation with (Cd, Zn, S, Se) shells have increased the photoluminescence (PL) quantum yield (QY) up to almost unity and their narrow emission band can be tuned over the entire visible spectrum. For applications in which Cd-containing nanocrystals are restricted by an EU RoHS directive, InP QDs form a believable alternative. Recently, synthesis methods for colloidal InP nanocrystals have been developed on the basis of cheap and safe In- and P-precursors, which allow the production of NCs on a large scale. ${ }^{1-3}$ Furthermore, suitable inorganic shells that contain $\mathrm{Zn}, \mathrm{Cd}, \mathrm{S}$, and $\mathrm{Se}$ have been developed that increased the PL QY from nearly zero to values up to $60 \%{ }^{4}$ This would enable applications of InP QDs as labels in biology, as phosphors in large-scale lighting, lasers and quantum optics. ${ }^{5-10}$ Nevertheless, experimental studies on the optical properties of colloidal InP QDs remain scarce and the understanding of their PL properties has remained limited. For example, the origin of the emission lines observed in Fluorescence Line Narrowing (FLN) spectra of InP QDs have remained unexplained for decades. ${ }^{11}$ Furthermore, the properties of the dark exciton state (spin dynamics, recombination mechanism, activation mechanism) have not been unveiled, while they are of paramount importance in the perspective of using colloidal InP QDs in spin-based applications such as memories, Q-bit or to design spin-preserving environment.

In zinc blende (ZB) or wurtzite (WZ) CdSe and ZB CdTe colloidal nanostructures, the lowest energy exciton (eightfold degenerate) is split into five fine structure levels by the electronhole exchange interaction, the intrinsic crystal field and/or the crystal shape anisotropy. ${ }^{12}$ The resulting exciton fine structure levels are characterized by their exciton total angular momentum projection, $\mathrm{F}=\mathrm{S}+\mathrm{M}$ where $\mathrm{S}$ is the electron spin and $\mathrm{M}$ the angular momentum of the hole. The lowest exciton level is a $F= \pm 2$ state (which is dipole forbidden for direct radiative recombination, thus optically dark), followed by higher-energy exciton states, with $\mathrm{F}$ $= \pm 1$ (which are dipole allowed, thus optically bright), and with $\mathrm{F}=0$ (one optically dark and one optically bright). ${ }^{12}$ For crystals with $\mathrm{ZB}$ or WZ lattice, a magnetic field parallel to the crystal or to the shape symmetry axis (called q-axis) lifts the exciton spin degeneracy into Zeeman levels with projection $\mathrm{F}=-2$ and $\mathrm{F}=+2$ for the dark state, and $\mathrm{F}=-1$ and $\mathrm{F}=+1$ for the bright state. For B perpendicular to q-axis, the dark and bright exciton are mixed, so the 
dark excitons gain oscillator strength from the bright excitons. ${ }^{12}$ Once drop casted on a substrate, spherical QDs have their q-axis randomly oriented with respect to the magnetic field; this means that Zeeman splitting as well as bright-dark mixing should be observed in ensemble measurements under high magnetic field. Thorough studies performed on CdSe QDs have allowed unveiling the experimental fingerprints of each configuration in ensemble measurements. Namely, the Zeeman interactions lift the spin degeneracy of the fine structure states which leads to a splitting of the emission lines in PL spectra as well as a build-up of a spin population (up or down depending on the sign of the electron and hole g-factors). ${ }^{13,14}$ The spin accumulation is clearly evidenced by the magnetic field-induced circular polarization of the PL emission. ${ }^{13-16}$ By contrast the smoking gun of the bright-dark mixing at cryogenic temperature arises from the non-polarized PL properties in magnetic field. In Fluorescence Line Narrowing (FLN) and single dot PL spectra, a strong enhancement of the intensity of the Zero Phonon Line (ZPL), which is attributed to the dark exciton recombination, was observed with increasing magnetic field due to the bright-dark exciton mixing. ${ }^{12,16-18}$ In addition to these spectral properties, the magnetic field-induced bright-dark mixing is unambiguously evidenced in PL decay measurement. At $\mathrm{B}=0 \mathrm{~T}$, the PL decays at cryogenic temperature occur on two markedly different timescales where the fast sub-nanosecond time stems from the bright-to-dark spin-flip limited radiative recombination of the bright exciton and the long living component from the radiative recombination of the dark exciton. ${ }^{19,20}$ Applying an external magnetic field induces a shortening of the long component, which eventually converges towards the bright exciton radiative lifetime. To date these fingerprints have been observed in many colloidal nanostructures: $\mathrm{CdSe},{ }^{21} \mathrm{CdSe} / \mathrm{ZnS},{ }^{17,18} \mathrm{CdSe} / \mathrm{CdS}$ (dot-in-rod, ${ }^{13,22}$ spherical with thin shell $\left.{ }^{14,16}\right)$, CdSe nanoplatelets, ${ }^{23,24}$ spherical $\mathrm{CdTe}^{25}$ Lead-halide perovskite. $^{26,27}$

\section{RESULTS}

Here we report the study of the time-, spectral- and polarization- resolved optical properties of ensemble of core/shell InP-based QDs having various core sizes and shell nature under high magnetic fields up to $30 \mathrm{~T}$. These thorough studies allow for unveiling the origin of the spectral lines in FLN spectra that have remained unexplained for decades. Notably we show that the lack of ZPL indicates a radiative recombination of the dark exciton that is mostly achieved by phonon-assisted process. This result is in marked contrast with the typical FLN spectra of any other colloidal nanostructures where strong ZPL signal is observed. Strikingly, our results evidence i) a lack of field-induced mixing between the bright and the dark states 
up to $30 \mathrm{~T}$, ii) a spin relaxation time comparable to the dark exciton lifetime $(\sim 1 \mu \mathrm{s})$, iii) as well as PL decay lengthening with increasing magnetic field. Importantly these features do not depend on the sample characteristics (core size, nature of the shell). Interestingly the lengthening of the PL decay in magnetic field points to a strong interaction between the exciton and magnetic moments within InP NCs. More specifically, our results show that the interaction of the spin of the electron within the dark exciton interacts with nuclear spins rather than with dangling bond spins at the core/shell interface. Interestingly, the interaction with nuclear spins vanishes upon applying a magnetic field because the energy conservation rule cannot be fulfilled. These results shed light on the potential use of colloidal InP nanocrystals in spin-based applications.

We studied $5 \mathrm{InP} / \mathrm{ZnS}$ samples that differ by their InP core size which diameter varies from $2.5 \mathrm{~nm}$ to $3.5 \mathrm{~nm}$ up to $15 \mathrm{~T}$ (for details see in $\mathrm{ref}^{20}$ ) and two $\mathrm{InP} / \mathrm{ZnS}$ samples up to $30 \mathrm{~T}$ (see methods). For comparison, the results obtained with other InP core/shell samples, with shells composed of $\mathrm{ZnSe}$ or $\mathrm{Cd}_{0.09} \mathrm{Zn}_{0.91} \mathrm{Se}$ alloy (Supporting Information, Figure S1). Due to the zinc blende crystal structure and the prolate shape of the InP cores, it can be expected that $\mathrm{InP} / \mathrm{ZnS}$ nanocrystals will show an exciton fine structure comparable to that measured with CdSe-based QDs, with a dark ground state exciton that dominates the optical properties at cryogenic temperature. By contrast with CdSe QDs, the PL spectra of InP/ZnS QDs are complex since photons stemming from upper excitonic states, laying $100 \mathrm{meV}$ above the bright-dark doublet, participate to the PL signal. ${ }^{20}$ Therefore, special care should be taken when studying magnetic field-induced optical properties of InP/ZnS QDs. To overcome the limitation of ensemble broad featureless PL spectra, it is convenient to use Fluorescence Line Narrowing (FLN) techniques that lead to emission lines with a FWHM about one order of magnitude sharper. In FLN experiments, the closest line to the laser is commonly referred to as the zero phonon line (ZPL). However, this line is rather composed of a sharp ZPL line together with acoustic phonon sideband. ${ }^{28}$ The FLN spectra obtained on core/shell InP-based colloidal QDs (Figure 1a) are very similar to those observed by Micic et al. who studied InP QDs passivated with hydrogen fluoride. The FLN spectra are composed of two main peaks shifted from the excitation energy by about 25 and $58 \mathrm{meV} .{ }^{11}$ By analogy with CdSe, the first peak, shifted by $25 \mathrm{meV}$, was attributed by Micic et al. to the ZPL from the dark state. So its shift from the laser was taken as a reference for the bright-dark splitting, $\Delta \mathrm{E}_{\mathrm{AF} .}{ }^{11}$ However from our previous report, we obtained significantly lower bright-dark splitting $(<15 \mathrm{meV})$ from the temperature dependence of the PL decay. ${ }^{20}$ Therefore, neither lines on InP FLN 
spectra have an energy shift from the laser line that corresponds either to the LO phonon line in bulk InP (43 meV) or to the bright-dark energy splitting. ${ }^{20}$ It should be noted that the value of the LO phonon energy in InP QD has been verified by Raman spectroscopy and corresponds to the bulk value $(\sim 43 \mathrm{meV}) .{ }^{29}$ Therefore in our analysis, we assume that the peak at around $58 \mathrm{meV}$ represents the LO phonon replica of the dark state emission. The bright-dark splitting is then given by the relative position of the ZPL to the laser line (Figure 1b). Here, we find a bright-dark splitting of $15 \mathrm{meV}$ in agreement with previous measurement. ${ }^{20}$ Finally, the main PL line at $\sim 25 \mathrm{meV}$ is attributed to the acoustic phonon sideband (Ac) in agreement with a recent study on InP/ZnSe QDs. ${ }^{30}$ This attribution allows for solving the apparent controversy in the bright-dark energy splitting obtained from FLN spectra and from the temperature dependence of the PL decay. This finding strongly suggests that, in marked contrast with CdSe QDs, the radiative recombination of the dark exciton in InP QDs is almost exclusively phonon-assisted and the relative weight of the ZPL in the PL spectra is negligible. This difference is schematically illustrated in Figure $1 \mathrm{~b}$ and c. The magnetic field dependence of unpolarized FLN spectra strongly support these results. Strikingly, Figure 1a shows that the FLN spectra hardly depend on the magnetic field strength up to $30 \mathrm{~T}$ (see also Supporting Information, Figure S2). The slight increase of the peaks position stems from the Zeeman splitting. By contrast with CdSe QDs, the intensity of the first PL line is not enhanced upon rising the magnetic field and its intensity remains constant (inset of Figure 1a, the change in intensity of the main peak is less than $10 \%$ ). These striking results, observed on the InP/ZnS QDs studied here suggest an absence of magnetic field induced bright-dark mixing and confirm our previous assignment of the emission line to acoustic and optical phonon replica. We emphasize that the absence of bright-dark mixing has only been reported at the single dot level ${ }^{17,18}$ and in epitaxially grown QDs. ${ }^{31}$ 


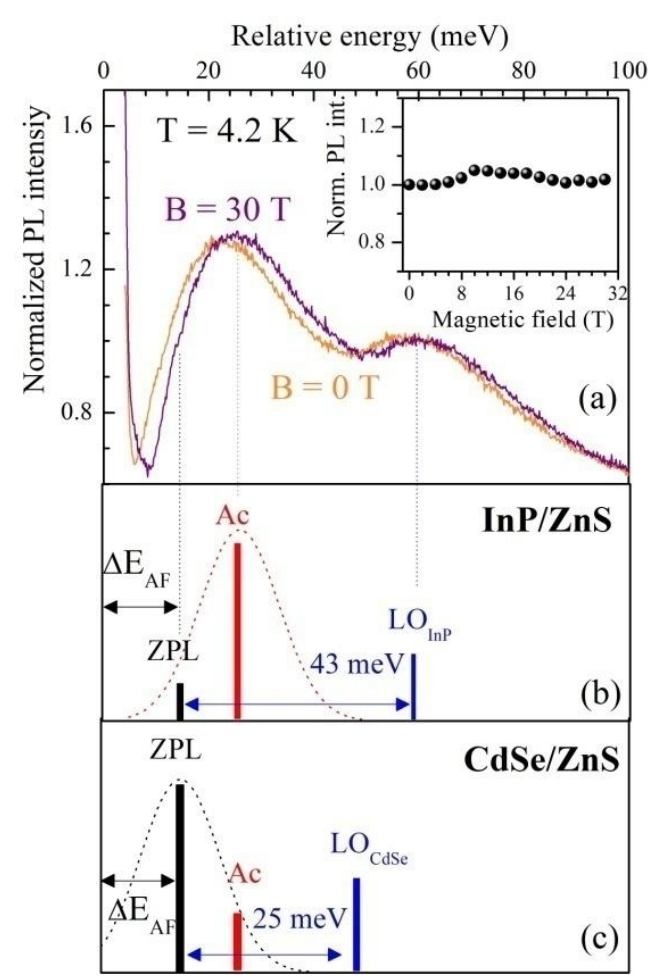

Figure 1 a) FLN spectra of InP/ZnS nanocrystals with $2.8 \mathrm{~nm}$ diameter at $T=4.2 \mathrm{~K}$ and $B=0 \mathrm{~T}$ (orange) and $30 \mathrm{~T}$ (purple). The sample is resonantly excited with laser at $2.33 \mathrm{eV}$. Inset: Magnetic field dependence of the normalized integrated PL intensity. b) Model of the FLN spectra in InP/ZnS. The lowest energy peak corresponds to the 1 LO line and is shifted from the ZPL of the dark exciton by $43 \mathrm{meV}$. The ZPL is shifted from the laser line by the bright dark splitting of $15 \mathrm{meV}$. The main peak is attributed to the acoustic phonon sideband. c) Model of the FLN spectra in CdSe nanocrystals. The first peak is dominated by the ZPL of the dark exciton and is separated by $25 \mathrm{meV}$ from the first LO replica.

The lack of mixing is further confirmed by the nearly constant unpolarized PL decay with increasing magnetic field (Figure 2a). Strikingly, the PL decay remains constant around 1100 ns from 1-2 $\mathrm{T}$ to $15 \mathrm{~T}$ and even up to $30 \mathrm{~T}$ (Supporting Information, Figure S3). The lengthening from $850 \mathrm{~ns}$ to $1100 \mathrm{~ns}$ upon applying a magnetic field from 0T to 1-2 $\mathrm{T}$ will be discussed later. We must stress that a constant PL decay is only expected in the case of a magnetic field applied strictly parallel to the quantization axis since the bright and the dark exciton states are not mixed. In the case of single CdSe NCs, it was shown that an angle of $25^{\circ}$ between the magnetic field and the c-axis of the nanocrystal is sufficient to induce a bright-dark mixing. ${ }^{17}$ Instead we observe a complete absence of shortening of the PL decay for all the InP/ZnS QDs in ensemble measurement (see Supporting Information, Figure S4) as well as in InP QDs with ZnSe and CdZnSe shells (Supporting Informations, Figure S5). Therefore, the optical properties of InP/ZnS QDs in magnetic field are mostly compelled by 
the Zeeman interaction. These results allow for studying the magnetic field-induced polarization, the spin dynamics and the g-factors of excitonic states on NCs ensembles.
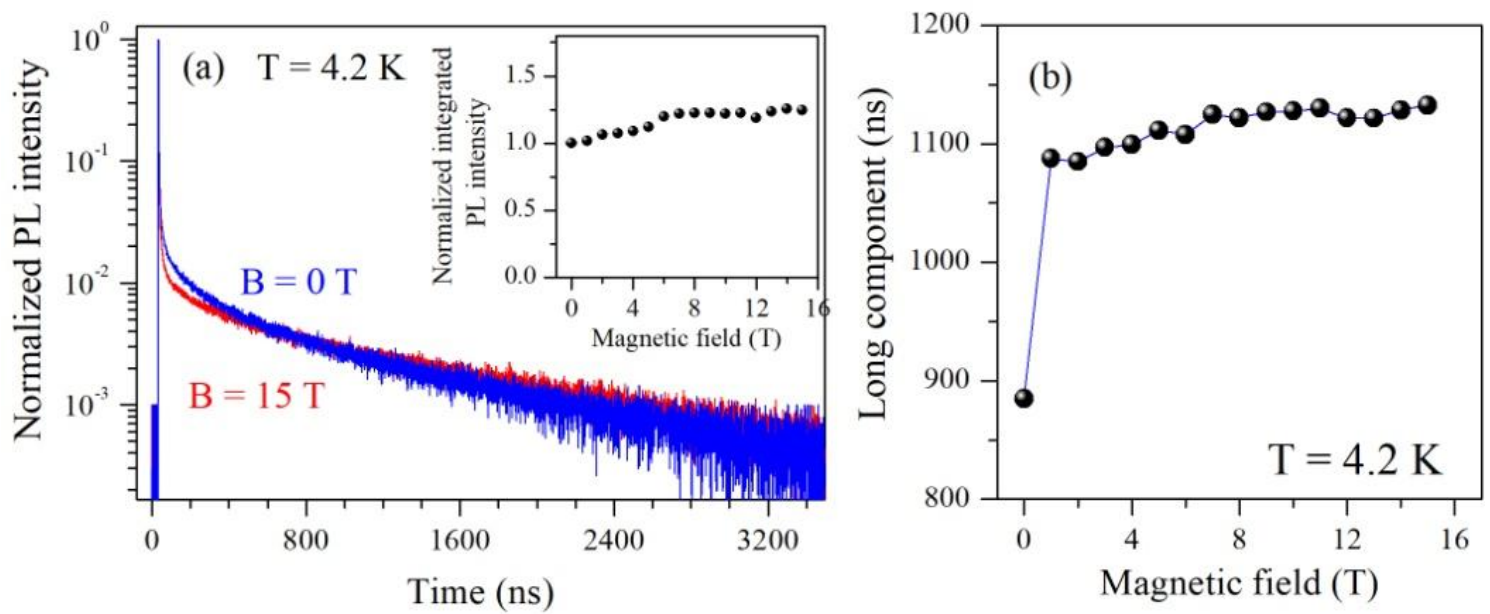

Figure 2: a) Unpolarized normalized PL decay at $B=0 T$ and $15 T$ at $T=4.2 \mathrm{~K}$ of ensemble of InP/ZnS nanocrystals with $2.9 \mathrm{~nm}$ core diameter filtered around the ensemble PL maximum $(E=2.1$ $\mathrm{eV}$ ) and excited non resonantly at $3.0 \mathrm{eV}$. Inset: Magnetic field dependence of the normalized integrated PL intensity. b) Long component of the PL decay as a function of the magnetic field. Solid line is guide for the eyes.

Figure 3a shows the polarization-resolved PL spectra of the same InP/ZnS sample at a magnetic field of $15 \mathrm{~T}$ together with the spectrally resolved Degree of Circular Polarization (DCP). The DCP, $P_{C}$, is defined as:

$$
P_{C}=\frac{I^{+}-I^{-}}{I^{+}+I^{-}}=\frac{\tau_{R}}{\tau_{R}+\tau_{s}} \tanh \left(\frac{g \mu_{B} B}{2 k_{B} T}\right),
$$

where $I^{+}\left(I^{-}\right)$corresponds to the intensity of the spin up (down) polarization. $\tau_{\mathrm{R}}$ and $\tau_{\mathrm{S}}$ are the exciton lifetime and spin relaxation time, g the Landé g-factor. The DCP is negative, which indicates a dominant contribution of $\sigma$ - polarization in magnetic field. ${ }^{13-16,26}$ By contrast with CdSe NCs we observe a strong spectral dependence of the DCP. The DCP in the high energy part of the PL spectra is twice larger than the DCP in the low energy part (Figure 3a). In a previous work, we demonstrated that high energy photons stem from the spin-relaxation limited radiative recombination of excitonic states lying about $100 \mathrm{meV}$ above the bright-dark doublet (Figure 3b). ${ }^{20}$ Therefore the large values of DCP results from the spin relaxation of the upper bright states whereas the low values correspond to the spin relaxation of the brightdark doublet. 
Figure $3 \mathrm{c}$ shows the magnetic field dependence of the DCP at high and low energy of the PL spectrum, where solely upper bright and dark exciton contributes, respectively. Surprisingly, in Figure 3c, the magnetic field dependence of the time-integrated DCP show saturation values around $-0.35(-0.16)$ at $\mathrm{B}=15 \mathrm{~T}$ for the high (low) energy part of the PL spectrum. It is noteworthy that a saturation around -1 could be expected in the framework of pure Zeeman interactions of spherical zinc blend nanocrystals and of -0.75 for random orientation of q-axis in ensemble of QDs. These results are in agreement with the spectrally-integrated DCP of InP/ZnS measured up to $30 \mathrm{~T}$, and of InP QDs with ZnSe and CdZnSe shell. (Supporting Information, Figures S6 and S7).

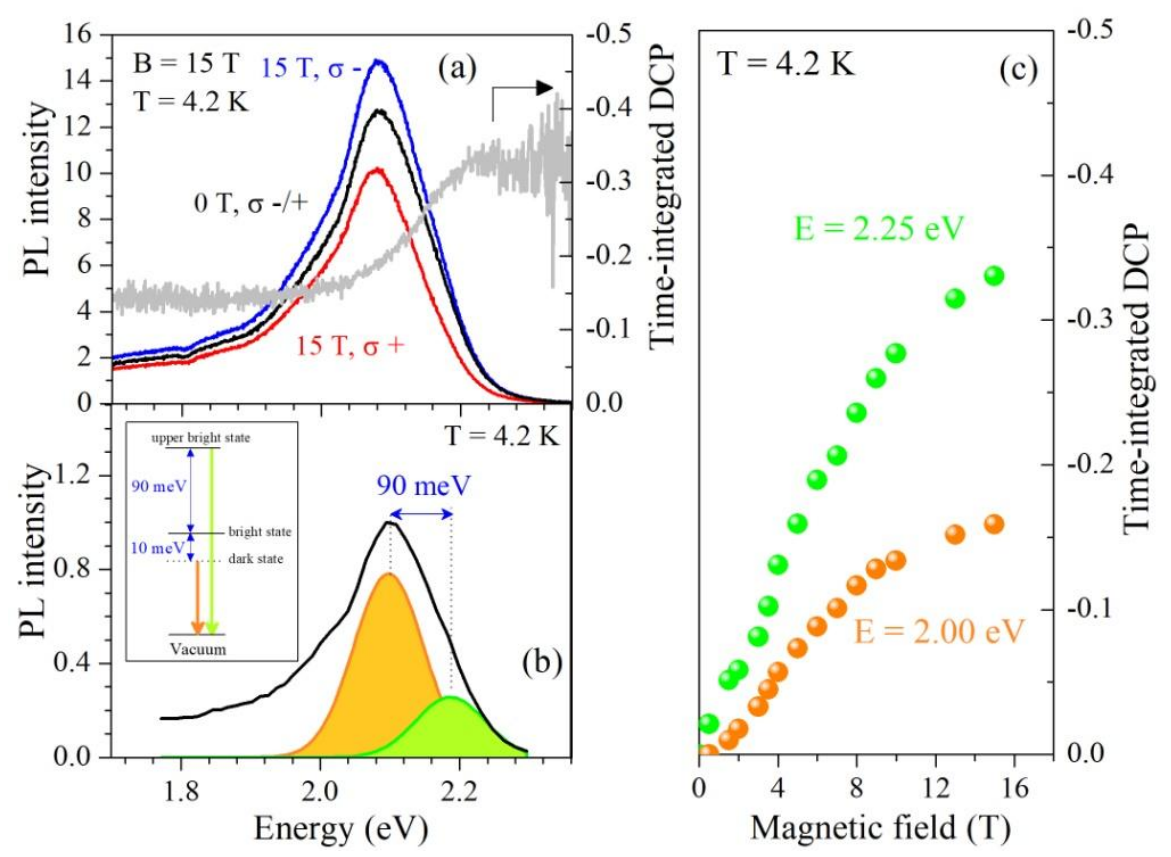

Figure 3 a) Polarization resolved PL spectra of InP/ZnS NCs with $2.9 \mathrm{~nm}$ core diameter at $T=4.2 \mathrm{~K}$ for magnetic field $B=O T$ and $15 T$ together with the spectrally resolved DCP (light grey). Laser excitation at $3.0 \mathrm{eV}$. b) Same PL spectrum at OT (black) with the highlighted contributions to the PL spectrum of the upper bright state (green) and the bright-dark doublet (orange). Inset: detailed exciton fine structure involved in the PL emission (adapted from Ref ${ }^{20}$ ). c) Magnetic field dependence of the time-integrated DCP at the spectral position of the upper bright state (green) and the brightdark doublet (orange).

The non-linear rise of the DCP between $\mathrm{B}=0 \mathrm{~T}$ and $8 \mathrm{~T}$ (Figure 3c) indicates that the spin relaxation time $\tau_{\mathrm{S}}$, between the Zeeman sublevels must be comparable to the lifetime of the exciton, $\tau_{\mathrm{R}} \cdot{ }^{14,32}$ In order to get deeper insight into the spin dynamics, we studied time-resolved degree of circular polarization (TRDCP). Figure 4a shows the polarization-resolved PL decay at $15 \mathrm{~T}$ at the PL maximum of the same $\mathrm{InP} / \mathrm{ZnS}$ sample $\left(\mathrm{E}_{\mathrm{det}}=2.1 \mathrm{eV}\right)$ together with the corresponding TRDCP. We clearly see that the buildup of the spin polarization occurs on two 
markedly different time scales. The fast initial rise occurs in $24 \mathrm{~ns}$ while the relaxation to the saturation levels $\mathrm{P}_{\mathrm{sat}}=-0.27$ is achieved in $400 \mathrm{~ns}$. The origin of the spin dynamics can hardly be unveiled from the TRDCP at the PL maximum since bright, dark and higher exciton states (Figure $3 b$ ) contribute to the PL signal. ${ }^{20}$
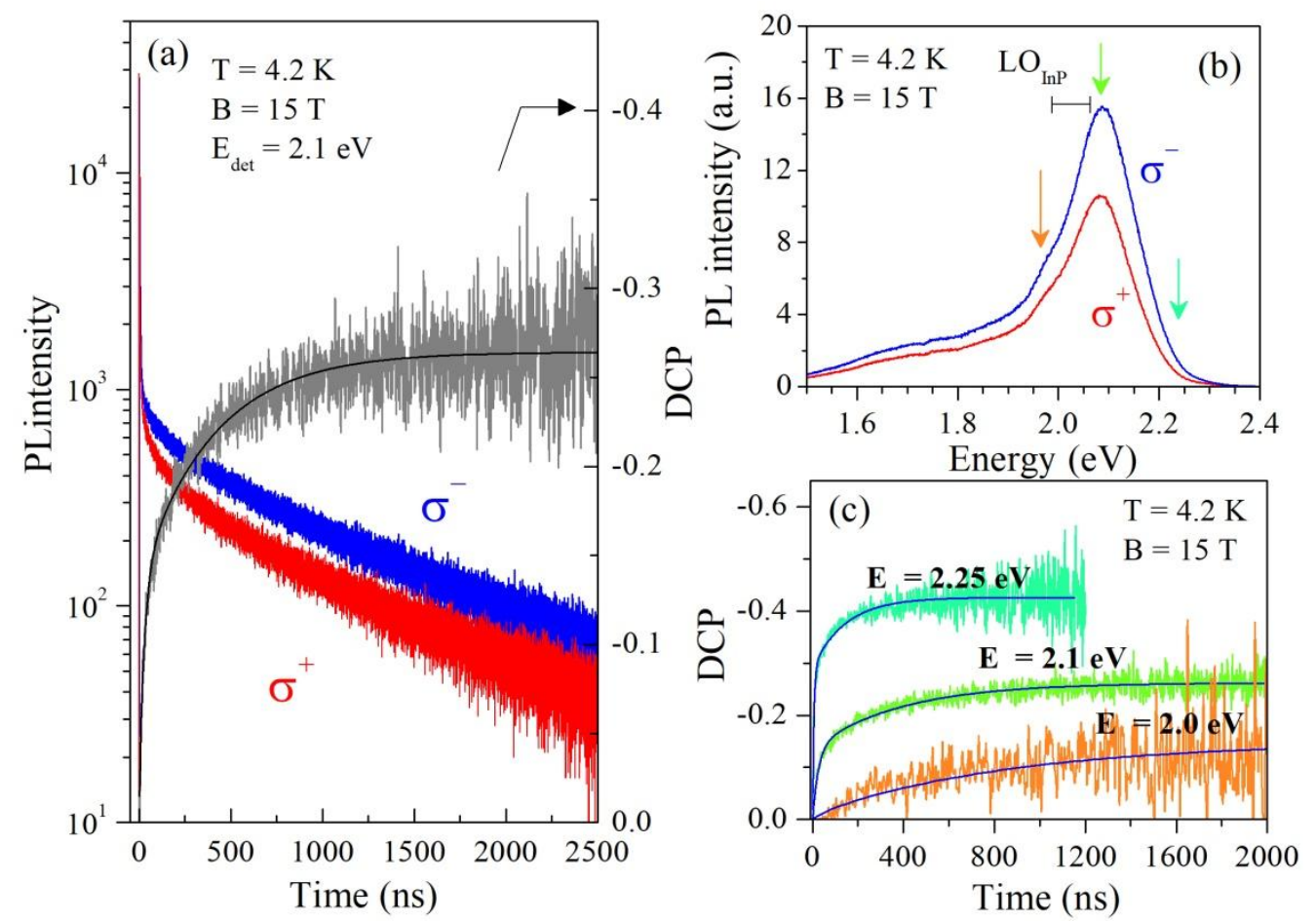

Figure 4: a) Time-resolved PL decay at the maximum of the $P L(E=2.1 \mathrm{eV})$ (green arrow in panel b) of ensemble of InP/ZnS (same as Figure 3) at $T=4.2 \mathrm{~K}$ and $B=15 T$ of $\sigma$ - (blue) and $\sigma+($ red) polarization together with the time-resolved DCP (grey). Solid line is a two exponential fit with times $\tau_{\text {short }}=24 \mathrm{~ns}$ and $\tau_{\text {long }}=400 \mathrm{~ns}$. b) Corresponding polarization resolved PL spectra. c) Time-resolved DCP at spectral position indicated by arrows in panel $b$.

The spectral dependence of the TRDCP allows to separate the various contributions: the low energy part of the spectrum results only from the radiative recombination of the dark exciton (Figure $4 \mathrm{~b}$, orange arrow at $2.00 \mathrm{eV}$ ), while the high energy part mostly results from the radiative recombination of higher bright state (Figure $4 \mathrm{~b}$, blue arrow at $2.25 \mathrm{eV}$ ). The TRDCP measured at $\mathrm{E}=2.00 \mathrm{eV}$ (Figure 4c) corresponds to the spin dynamics of the dark ground exciton, which can be well reproduced by a single exponential function with a spin relaxation time of $\sim 400 \mathrm{~ns}$. This is several orders of magnitude longer than in ensemble of CdSe-based QDs. ${ }^{14,15}$ The slow spin dynamics can, in principle, be due to the spin relaxation between the dark exciton Zeeman levels which requires a simultaneous spin flip of the electron and the 
hole. ${ }^{17}$ We emphasize that the slow spin dynamics observed for InP QDs is in line with the absence of magnetic field induced bright-dark mixing. This is in firm contrast to the case of CdSe QDs where a fast spin relaxation time goes hand-in-hand with bright-dark mixing. ${ }^{13}$ The dark exciton spin relaxation time $\left(\tau_{S}=\sim 400 \mathrm{~ns}\right)$ is comparable to its radiative recombination time $\left(\tau_{R}=1100 \mathrm{~ns}\right)$, which implies a significant contribution of the dynamical factor, $\rho=\tau_{R}$ $/\left(\tau_{R}+\tau_{S}\right)$, in the DCP value even at fields up to $15 \mathrm{~T}(\rho=0.73)$.

By contrast, the TRDCP at high energy $(\mathrm{E}=2.25 \mathrm{eV})$ shows that the spin thermalization is achieved mostly within $6 \mathrm{~ns}$. We attribute this fast spin dynamic to the relaxation from the upper bright state to lower exciton states, which requires only one charge carrier spin-flip, rather than in-between Zeeman levels that requires a simultaneous spin-flip of both charge carriers. Note that the remaining long component in the TRDCP is likely due to residual contribution of the dark exciton line (cf Figure 3b). It is noteworthy that this inter level spinflip time of $\sim 6 \mathrm{~ns}$ is significantly longer under magnetic field than those previously reported at $0 \mathrm{~T}$ where a spin relaxation time of 700 ps was measured. ${ }^{20}$

\section{DISCUSSION}

Absence of magnetic field induced bright-dark mixing. Above, we presented results that show that a magnetic field does not mix the spin-forbidden dark and bright states in InP QDs with $\mathrm{ZnS}, \mathrm{ZnSe}$ or $(\mathrm{Zn}, \mathrm{Cd}) \mathrm{Se}$ shells. We emphasize that recent results obtained on InP/ZnSe QDs support the lack of bright dark mixing. ${ }^{30}$ The authors could not observe the fingerprint of the bright-dark mixing. Instead they extracted, for various InP core size, a nearly constant bright exciton g-factor $\left(g_{B}=3.5\right)$ from the spectral shift of the PL line with magnetic field. Importantly this value corresponds to the bright exciton g-factor value previously measured on colloidal InP/ZnS nanocrystals ${ }^{33}$ and on wurtzite InP nanowires when B // q-axis. ${ }^{34}$ This situation is rather unusual, and in strong contrast with, for instance, CdSe-based QDs. According to the existing theory, bright-dark mixing is absent when the magnetic field is parallel to a high-symmetry axis. ${ }^{12}$ Note that in this framework, the dark and the bright exciton state are respectively attributed to the states $| \pm 2\rangle$ and $\left| \pm 1^{\mathrm{L}}\right\rangle$. Such a situation has occasionally been observed in single-dot photoluminescence spectroscopy. ${ }^{17}$ In QDs ensemble, the symmetry axes are randomly oriented and bright-dark mixing occur. ${ }^{13,15,25,35}$ Following commonly used k.p theory, the mixing appears because the off diagonal terms in the Hamiltonian are non-zero when the magnetic field is not strictly parallel to the q-axis. In this case, the only possibility to explain an absence of bright-dark mixing lays in the 
vanishing of the matrix element which couples the excitonic states. ${ }^{12}$ We emphasize that this mixing should depend on the bright-dark energy splitting and on the electron g-factor, thereby should be size dependent which is in marked contrast with our results. In fact, no magnetic field induced shortening of the dark exciton lifetime was observed for all the InP core size and for different shells (Supporting Information, Figure S4 and S5). This finding strongly suggest that the lack of bright dark mixing is rather due to an intrinsic property of $\mathrm{ZB}$ InP semiconductor.

Another possibility to explain the lack of shortening is to consider that the ground exciton is built from a $1 \mathrm{~S}$ electron and a $1 \mathrm{P}_{3 / 2}$ hole instead of a $1 \mathrm{~S}_{3 / 2}$ hole. ${ }^{36}$ In this $1 \mathrm{~S}_{\mathrm{e}} 1 \mathrm{P}_{3 / 2}$ exciton model, all the transition from the fine structure level are dipole forbidden. Therefore, the magnetic field will not affect the PL decay since all excitonic states are dark. Nevertheless this model fails to explain several experimental observations such as the possibility to perform FLN experiments which require a dipole allowed transition for the resonant excitation, or to explain the strong temperature dependence of the PL decay ${ }^{20}$ which points to the thermal population equilibration between 2 levels with strikingly different oscillator strength. Finally, the order between the $1 S_{3 / 2}$ and $1 \mathrm{P}_{3 / 2}$ hole level strongly depends on the NCs size. In particular, the $1 \mathrm{P}_{3 / 2}$ hole level is expected to be the ground hole state for small InP. Therefore, the switching between the $1 \mathrm{~S}_{3 / 2}$ and the $1 \mathrm{P}_{3 / 2}$ hole levels should be accompanied by a striking change in the optical properties in magnetic fields. However, our results obtained on InP NCs with core sizes below $2 \mathrm{~nm}$ up to $3.5 \mathrm{~nm}$ do not show this significant difference.

The only other possibility to explain the lack of bright-dark mixing is to consider that the exciton fine structure in InP QDs is weakly sensitive to shape anisotropy. In this case, the electron-hole exchange interaction splits the eightfold degenerate band-edge exciton into a fivefold degenerate dark exciton with total angular momentum 2 and a threefold degenerate bright exciton with total angular momentum $1 .{ }^{12}$ In this framework the degenerated bright and dark states are not coupled by the magnetic field. We emphasize that this situation can only occur for perfectly spherical ZB QDs or for anisotropic QDs where the anisotropy splitting vanishes. ${ }^{12}$ While the former situation is almost impossible to achieve at the ensemble level, the latter case might occur for zinc blend anisotropic QDs for which the light hole to heavy hole effective mass ratio, $\beta$, is about $0.14 .{ }^{12}$ We must stress that this value is very close to the calculated ratio for $\operatorname{InP}(\beta=0.148) .{ }^{37}$ This results strongly suggest that zinc blende InP-based QDs host nearly-isotropic excitons, regardless of the QD shape. 
Weak circular polarization degree induced by magnetic field. Compared to CdSe QDs, the saturation level of the DCP in InP/ZnS QDs is much lower, especially in the low energy part of the PL spectra, which is dominated by the dark exciton recombination. The low DCP values could be caused by several effects. First, a small dark exciton g-factor might prevent the buildup of a spin population since the resulting Zeeman splitting, $g \mu_{B} B$, is smaller than the thermal energy even at cryogenic temperatures. A long spin dynamic also hampers the buildup of a spin population. This contribution is reflected in the dynamical factor, $\tau_{R} /\left(\tau_{R}\right.$ $\left.+\tau_{S}\right)=0.7$. Here, assuming a saturation value of -1 for the DCP, the dynamical factor only contributes to lower the DCP down to -0.7 . Moreover, Rodina et al. showed that the dark exciton state can couple to upper bright exciton state $\left|0^{\mathrm{U}}\right\rangle$ by acoustic phonons. ${ }^{38}$ This coupling is notably responsible for the low DCP values in $\mathrm{CdSe} / \mathrm{CdS}$ dot-in-rod nanostructures. ${ }^{13,22}$ In the case of a lack of bright dark mixing and a dominant coupling to acoustic phonons, as observed in InP/ZnS QDs, the dark exciton polarization properties will be given by the $\mid 0^{\mathrm{U}}>$ state which shows linear polarization. This latter effect explains the low DCP values observed here in $\mathrm{InP} / \mathrm{ZnS}$ as well as in $\mathrm{InP} / \mathrm{ZnSe} \mathrm{QDs}^{30}$ and in a previous report by Langof et $a l^{33}$

Dark exciton lifetime lengthening with magnetic field. First we emphasize that this lengthening has been observed on all InP QDs, regardless the shell $\left(\mathrm{ZnS}, \mathrm{ZnSe}\right.$, or $\mathrm{Cd}_{0.09}$ $\mathrm{Zn}_{0.91} \mathrm{Se}$ ) or the InP core size. Such a lengthening implies the cancelling of a recombination mechanism of the dark exciton that is sensitive to an external magnetic field. Among the recombination pathways identified for the dark exciton, ${ }^{38}$ only the mechanisms involving flip-flop of the electron spin with a spin reservoir can be sensitive to the magnetic field. This mechanism is compelled by the spin conservation criterion which can be fulfilled by different means (Figure 5a). Typically, these spin interactions are described by the hyperfine interactions $(\mathrm{HF}){ }^{39,40}$ In the literature two main sources of spin reservoir have been identified: surface dangling bond spins (DBS) in II-VI colloidal nanocrystals ${ }^{17,41}$ and nuclear spins. ${ }^{42-44}$ Opposite from other nanostructures, all ions in InP have a magnetic moment and $\mathrm{InP} / \mathrm{ZnS}$ QDs have trap states at the interface, ${ }^{20}$ so that electrons in colloidal InP/ZnS QDs might interact with both reservoirs.

In the case of DBS reservoir, a magnetic field leads to the thermodynamic formation of a dangling bond magnetic polaron (DBMP) which prevents the electron spin flip-flop assisted mechanism to occur, ${ }^{41,45}$ thereby it induces a lengthening of the radiative recombination of the dark exciton (Figure 5b). ${ }^{17}$ By contrast the Zeeman splitting of nuclei spins is more than three 
orders of magnitude smaller than for the electron. Hence, the flip-flop mechanism between the spin of the electron and the nuclear spins in magnetic field is prevented because the energy conservation rule cannot be fulfilled (Figure 5c). The vanishing of this mechanism when the magnetic field is applied also leads to a lengthening of the dark exciton lifetime.
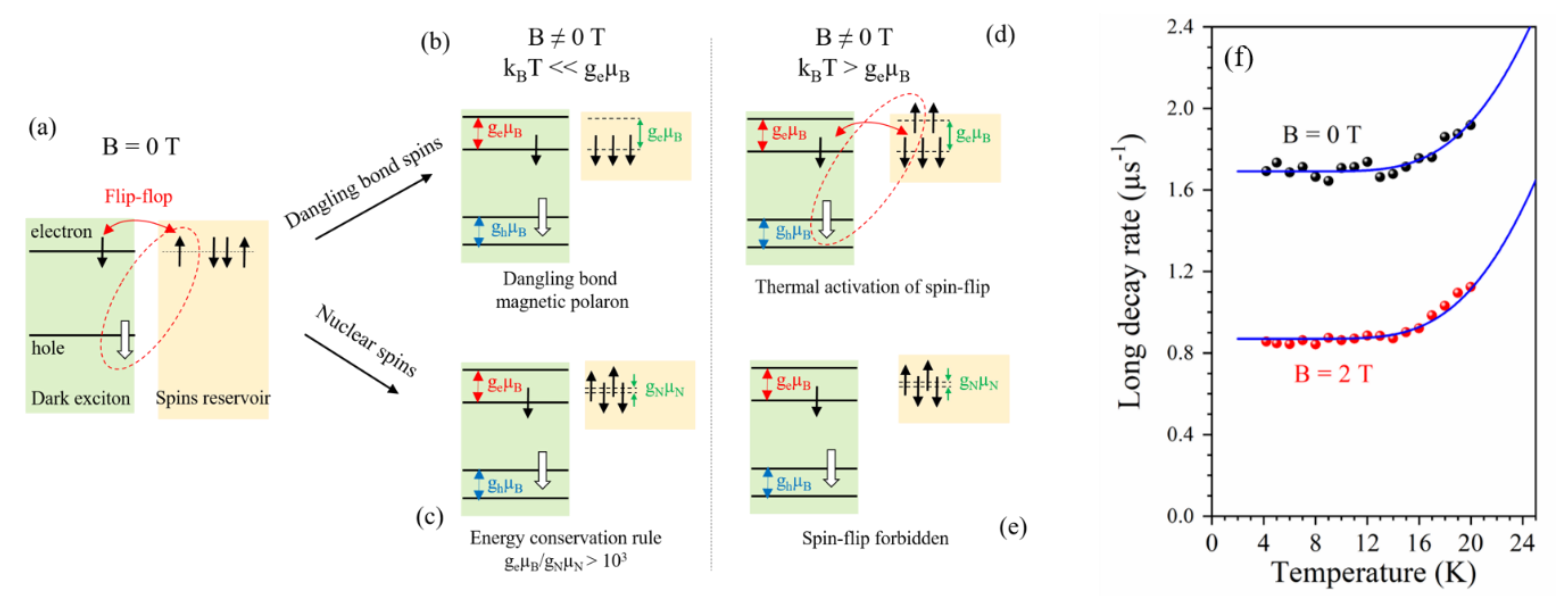

Figure 5: a) Dark exciton recombination activated by interaction between electron spin and a spin reservoir. b) The formation of a thermodynamic dangling bond magnetic polaron under magnetic field suppresses the spin-flip mechanism. c) The large energy mismatch in the Zeeman splitting of electron and nuclei breaks the energy conservation rule and prevents spin-flip mechanisms. d) For $k_{B} T>g_{e} \mu_{B} B$, the dangling bonds spins are randomized, which enables the spin-flip processes. e) The spin-flip process is inhibited by the energy conservation rule, regardless the thermal energy. f) Temperature dependence of long component of the PL decay at OT (black) and 2T (red) of ensemble of InP/ZnS NCs (2.9 nm core diameter) filtered around the PL maximum. Solid blue lines are fit (see ref ${ }^{20}$ ) where the bright exciton decay rate, $\Gamma_{A}=30 \mu s^{-1}$ and the bright-dark energy splitting, $\Delta E=8 \mathrm{meV}$ are fixed parameters and the dark exciton decay rate, $\Gamma_{F}$ is a fitting parameter. Best fit give $\Gamma_{F}=$ $0.86 \mu \mathrm{s}^{-1}$ for $B=2 \mathrm{~T}$ and $\Gamma_{F}=1.87 \mu \mathrm{s}^{-1}$ for $B=0 \mathrm{~T}$.

Therefore, at temperature such that $\mathrm{k}_{\mathrm{B}} \mathrm{T}<\mathrm{g}_{\mathrm{e}} \mu_{\mathrm{B}} \mathrm{B}$, both flip-flop mechanisms are cancelled by the magnetic field and their respective contribution cannot be disentangled. Given the intrinsic nature of both reservoirs, the situation can be clarified by rising the temperature such that $\mathrm{k}_{\mathrm{B}} \mathrm{T}$ $\geq \mathrm{g}_{\mathrm{e}} \mu_{\mathrm{B}} \mathrm{B}$. In this case, the spin-flip between electrons and nuclei remains forbidden under magnetic field, regardless the temperature (Figure 5e). So the long decay rate of the PL decay with and without magnetic field will displays a similar temperature dependence. Namely, the dark exciton recombination rate is temperature independent. By contrast, the thermal energy randomizes DB spins which reactivates the flip-flop mechanism with the electron spins 
(Figure 5d). ${ }^{41}$ It was shown that this mechanism induces a strong temperature dependence of the dark exciton recombination rate even for thermal energy smaller than the bright-dark splitting.

In Figure 5f we unambiguously show that the long component of PL decay, $\Gamma_{\mathrm{L}}$, at $\mathrm{B}=0 \mathrm{~T}$ and $2 \mathrm{~T}$ follow the same temperature dependence. Both were simulated using the standard brightdark exciton model ${ }^{19}$ with the same set of parameters (bright exciton radiative lifetime and the bright-dark splitting) ${ }^{20}$ except for the dark exciton radiative lifetime. The lack of temperature dependence of the long decay rate excludes a dominant contribution of dangling bondsassisted mechanism in the radiative recombination of the dark exciton. This result shows that in InP/ZnS QDs, the spin of the electron within the dark exciton is efficiently coupled to the nuclear spin bath. This flip-flop mechanism, that enables the radiative recombination of the dark exciton at $0 \mathrm{~T}$, is cancelled once an external magnetic field is applied. We must stress that this finding is supported by the magnetic field dependence of the Zeeman splitting observed in InP/ZnSe QDs which shows characteristic features of $\mathrm{HF}$ interactions: the nonlinear rise of the Zeeman splitting at weak magnetic field due to an Overhauser field. ${ }^{46}$ These striking properties might affect the spin coherence in InP QDs but might further be used to optically probe local magnetic field.

\section{CONCLUSION}

The optical properties of InP/ZnS QDs under magnetic field show a marked difference compared to II-VI-based colloidal nanostructures. The weak ZPL in FLN spectra indicates that in InP/ZnS QDs the radiative recombination of the dark exciton is mostly achieved by phonon assisted mechanism with a dominant contribution of acoustic phonons. Moreover, our results demonstrate an absence of bright-dark mixing induced by magnetic field up to $30 \mathrm{~T}$ which points to an anisotropy-tolerance of InP. This striking situation on QDs ensemble allows investigating in details the mechanism involved in the dark exciton recombination and the spin dynamics. In particular we show a long spin dynamics, comparable to the dark exciton radiative lifetime. We further evidence that in colloidal InP QDs, hyperfine interactions between the electron spin and the nuclear spins at 0T enable the radiative recombination of the dark exciton. This work highlight the richness of the spin physics in III$\mathrm{V}$ colloidal nanostructures. We believe this work will stimulate theoretical work on the optical and electronic properties of InP-based colloidal nanostructures. In addition, our results will 
undoubtedly lead to further investigations on the possibilities to manipulate and store spins in III-V colloidal materials.

\section{METHODS}

Sample preparation. InP/ZnS core/shell QDs samples were synthesized, following the method reported in $\mathrm{ref}^{20}$. $\mathrm{InP} / \mathrm{ZnSe}$ and $\mathrm{InP} /(\mathrm{Zn}, \mathrm{Cd}) \mathrm{Se}$ core/shell QDs samples were synthesized, following the method reported in ref.,47 The QD samples were washed by precipitation with methanol, isolated by centrifugation and redispersed in octadecene. To achieve a QDs film, the solution was deposited using a drop-cast method, on crystalline silicon substrate for FLN measurements, and on a quartz substrate for PL and TrPL measurements.

PL and Time-resolved Photoluminescence (TrPL). The Time-resolved PL (TrPL) and polarized PL measurements were performed on QD ensemble dropcasted on a quartz substrate. In the Time-resolved PL measurements the excitation was provided by a picosecond pulsed diode-laser operating at 405nm (pulse width $100 \mathrm{ps).} \mathrm{To} \mathrm{ensure} \mathrm{the} \mathrm{complete} \mathrm{decay} \mathrm{of}$ the excitonic population, a repetition rate of the laser below $200 \mathrm{kHz}$, controlled by the laser driver internal clock, was kept for all samples. The average count rate was kept below $2 \%$ of the laser repetition rate. This low excitation fluence was used in order to maintain single photon statistics and avoid multiexciton formation. The TrPL signal of the QDs ensemble was either spectrally integrated on all the wavelengths (Nijmegen, field up to 30T) or spectrally selected through a monochromator (Dortmund, field up to $15 \mathrm{~T}$ ). The PL signal was detected by an avalanche photo diode connected to a single-photon counter (time-correlated single photon counting).

Polarized PL measurements were performed in static (and time resolved) configuration, using the same excitation source, in continuous or pulse wave mode. The excitation was circularly 
polarized with the use of a linear polarizer and a Babinet Soleil compensator. The PL light was guided through a single grating spectrometer (300 grooves/mm grating) and detected by a liquid nitrogen cooled charge couple device (CCD) in the static configuration and by an avalanche photo diode connected to a single-photon counter (time-correlated single photon counting) in the time resolved configuration. The emitted photons were detected in crossed and co-polarization relative to the laser polarization by using a linear polarizer and a lambdaquarter wave plate. Cut-off optical filters were used in excitation and detection, for both PL and TRPL experiments.

Fluorescence Line-Narrowing (FLN) measurements. For these experiments the samples used were QD in glass cuvettes or in films on crystalline silicon substrate (see sample preparation). The samples in film were mounted in a titanium sample holder on top of a threeaxis piezo-positioner. The FLN measurements were performed using a narrow excitation source, achieved by using a tunable jet-stream dye (Rhodamine 6G) laser. This monochromatic laser beam was circularly polarized by means of a linear polarizer and a Babinet-Soleil compensator. The laser beam was focused on the sample by a singlet lens (10 $\mathrm{mm}$ focal length). The same lens was used to collect the PL emission and direct it to the detection setup (Backscattering geometry). The samples and optical probe were mounted inside a liquid-helium bath cryostat. The FLN signal was guided through a $0.3 \mathrm{~m}$ long single grating spectrometer (300 grooves/mm grating) and detected by a liquid nitrogen cooled charge couple device (CCD). The emitted photons were detected in crossed polarization relative to the laser polarization by using a linear polarizer and a lambda-quarter wave plate. Cut-off optical filters were used in excitation and detection.

\section{ACKNOWLEDGMENTS}

We acknowledge the support from HFML-RU/FOM, a member of the European Magnetic Field Laboratory (EMFL). We acknowledge the financial support by the Deutsche Forschungsgemeinschaft in the frame of the ICRC TRR160 (project B1) and TRR142 (project B1) and by the Government of Russia (project number 14. Z50.31.0021, leading scientist M. Bayer). We acknowledge the support by the European Commission via the Marie-Sklodowska Curie action Phonsi (H2020-MSCA-ITN-642656). DV acknowledge funding by the ERC Advanced Grant "FIRSTSTEP” 692691.

\section{ASSOCIATED CONTENT}


Supporting Information Available: Absorption and emission spectra of $\mathrm{InP} / \mathrm{ZnS} \mathrm{NCs}$, Emission spectra of InP/ZnS, InP/ZnSe and InP/CdZnSe NCs. Fluorescence Line Narrowing spectra in magnetic field of InP/ZnS NCs, PL decay in magnetic up to $30 \mathrm{~T}$ of $\mathrm{InP} / \mathrm{ZnS}$, InP/ZnSe and InP/CdZnSe NCs, Magnetic field dependence of the long component of the PL decay for $5 \mathrm{InP} / \mathrm{ZnS} \mathrm{NCs}$ sample, DCP for field up to $30 \mathrm{~T}$ of $\mathrm{InP} / \mathrm{ZnS}, \mathrm{InP} / \mathrm{ZnSe}$ and InP/CdZnSe NCs. This material is available free of charge via the Internet at http://pubs.acs.org.

\section{REFERENCES}

(1) Tessier, M. D.; Dupont, D.; De Nolf, K.; De Roo, J.; Hens, Z. Economic and Size-Tunable Synthesis of $\mathrm{InP} / \mathrm{ZnE}(\mathrm{E}=\mathrm{S}, \mathrm{Se})$ Colloidal Quantum Dots. Chem. Mater. 2015, 27, 4893-4898.

(2) Tessier, M. D.; De Nolf, K.; Dupont, D.; Sinnaeve, D.; De Roo, J.; Hens, Z. Aminophosphines: A Double Role in the Synthesis of Colloidal Indium Phosphide Quantum Dots. J. Am. Chem. Soc. 2016, 138, 5923-5929.

(3) Song, W.-S.; Lee, H.-S.; Lee, J. C.; Jang, D. S.; Choi, Y.; Choi, M.; Yang, H. Amine-Derived Synthetic Approach to Color-Tunable InP/ZnS Quantum Dots with High Fluorescent Qualities. J. Nanoparticle Res. 2013, 15, 1750.

(4) Dupont, D.; Tessier, M. D.; Smet, P. F.; Hens, Z. Indium Phosphide-Based Quantum Dots with ShellEnhanced Absorption for Luminescent Down-Conversion. Adv. Mater. 2017, 29, 1700686.

(5) Samuel, I. Electrifying Quantum Dots for Lasers. Nat. Mater. 2018, 17, 9-10.

(6) Shen, H.; Cao, W.; Shewmon, N. T.; Yang, C.; Li, L. S.; Xue, J. High-Efficiency, Low Turn-on Voltage Blue-Violet Quantum-Dot-Based Light-Emitting Diodes. Nano Lett. 2015, 15, 1211-1216.

(7) Chen, O.; Wei, H.; Maurice, A.; Bawendi, M.; Reiss, P. Pure Colors from Core-shell Quantum Dots. MRS Bull. 2013, 38, 696-702.

(8) Bourzac, K. Quantum Dots Go on Display. Nature 2013, 493, 283.

(9) Choi, M. K.; Yang, J.; Kang, K.; Kim, D. C.; Choi, C.; Park, C.; Kim, S. J.; Chae, S. I.; Kim, T.-H.; Kim, J. H.; Hyeon T.; Kim, D-H. Wearable Red-green-blue Quantum Dot Light-Emitting Diode Array Using High-Resolution Intaglio Transfer Printing. Nat. Commun. 2015, 6, 7149.

(10) Yang, Y.; Zheng, Y.; Cao, W.; Titov, A.; Hyvonen, J.; Manders, J. R.; Xue, J.; Holloway, P. H.; Qian, L. High-Efficiency Light-Emitting Devices Based on Quantum Dots with Tailored Nanostructures. Nat. Photonics 2015, 9, 259-266.

(11) Mićić, O. I.; Cheong, H. M.; Fu, H.; Zunger, A.; Sprague, J. R.; Mascarenhas, A.; Nozik, A. J. SizeDependent Spectroscopy of InP Quantum Dots. J. Phys. Chem. B 1997, 5647, 4904-4912.

(12) Efros, A. L.; Rosen, M.; Kuno, M.; Nirmal, M.; Norris, D. J.; Bawendi, M. Band-Edge Exciton in Quantum Dots of Semiconductors with a Degenerate Valence Band: Dark and Bright Exciton States. Phys. Rev. B 1996, 54, 4843-4856.

(13) Siebers, B.; Biadala, L.; Yakovlev, D. R.; Rodina, A. V; Aubert, T.; Hens, Z.; Bayer, M. Exciton Spin Dynamics and Photoluminescence Polarization of CdSe/CdS Dot-in-Rod Nanocrystals in High Magnetic Fields. Phys. Rev. B 2015, 91,155304. 
(14) Liu, F.; Biadala, L.; Rodina, A. V.; Yakovlev, D. R.; Dunker, D.; Javaux, C.; Hermier, J.-P.; Efros, A. L.; Dubertret, B.; Bayer, M. Spin Dynamics of Negatively Charged Excitons in CdSe/CdS Colloidal Nanocrystals. Phys. Rev. B 2013, 88, 035302.

(15) Furis, M.; Hollingsworth, J. A.; Klimov, V. I.; Crooker, S. A. Time- and Polarization-Resolved Optical Spectroscopy of Colloidal CdSe Nanocrystal Quantum Dots in High Magnetic Fields. J. Phys. Chem. B 2005 109, 15332-15338.

(16) Wijnen, F. J. P.; Blokland, J. H.; Chin, P. T. K.; Christianen, P. C. M.; Maan, J. C. Competition between Zero-Phonon and Phonon-Assisted Luminescence in Colloidal CdSe Quantum Dots. Phys. Rev. B 2008, 78,235318 .

(17) Biadala, L.; Louyer, Y.; Tamarat, P.; Lounis, B. Band-Edge Exciton Fine Structure of Single CdSe/ZnS Nanocrystals in External Magnetic Fields. Phys. Rev. Lett. 2010, 105, 157402.

(18) Louyer, Y.; Biadala, L.; Trebbia, J.-B.; Fernée, M. J.; Tamarat, P.; Lounis, B. Efficient Biexciton Emission in Elongated CdSe/ZnS Nanocrystals. Nano Lett. 2011, 11, 4370-4375.

(19) Labeau, O.; Tamarat, P.; Lounis, B. Temperature Dependence of the Luminescence Lifetime of Single CdSe/ZnS Quantum Dots. Phys. Rev. Lett. 2003, 90, 257404.

(20) Biadala, L.; Siebers, B.; Beyazit, Y.; Tessier, M. D.; Dupont, D.; Hens, Z.; Yakovlev, D. R.; Bayer, M. Band-Edge Exciton Fine Structure and Recombination Dynamics in InP/ZnS Colloidal Nanocrystals. ACS Nano 2016,10, 3356-3364.

(21) Nirmal, M.; Norris, D. J.; Kuno, M.; Bawendi, M. G.; Efros, A. L.; Rosen, M. Observation of the "Dark Exciton" in CdSe Quantum Dots. Phys. Rev. Lett. 1995, 75, 3728-3731.

(22) Granados Del Águila, A.; Jha, B.; Pietra, F.; Groeneveld, E.; de Mello Donegá, C.; Maan, J. C.; Vanmaekelbergh, D.; Christianen, P. C. M. Observation of the Full Exciton and Phonon Fine Structure in CdSe/CdS Dot-in-Rod Heteronanocrystals. ACS Nano 2014, 8, 5921-5931.

(23) Biadala, L.; Liu, F.; Tessier, M. D.; Yakovlev, D. R.; Dubertret, B.; Bayer, M. Recombination Dynamics of Band Edge Excitons in Quasi-Two-Dimensional CdSe Nanoplatelets. Nano Lett. 2014, 14, 11341139.

(24) Shornikova, E. V.; Biadala, L.; Yakovlev, D. R.; Sapega, V. F.; Kusrayev, Y. G.; Mitioglu, A. A.; Ballottin, M. V.; Christianen, P. C. M.; Belykh, V. V.; Kochiev, M. V.; Sibeldin, Nikolai N.; Golovatenko, A. A.; Rodina, A. V.; Gippius, N. A.; Kuntzmann, A.; Jiang, Y.; Nasilowski, M.; Dubertret, B.; Bayer, M. Addressing the Exciton Fine Structure in Colloidal Nanocrystals: The Case of CdSe Nanoplatelets. Nanoscale 2018, 10, 646-656.

(25) Blokland, J.; Claessen, V.; Wijnen, F.; Groeneveld, E.; De Mello Donegá, C.; Vanmaekelbergh, D.; Meijerink, a; Maan, J.; Christianen, P. Exciton Lifetimes of CdTe Nanocrystal Quantum Dots in High Magnetic Fields. Phys. Rev. B 2010, 83, 1-9.

(26) Canneson, D.; Shornikova, E. V.; Yakovlev, D. R.; Rogge, T.; Mitioglu, A. A.; Ballottin, M. V.; Christianen, P. C. M.; Lhuillier, E.; Bayer, M.; Biadala, L. Negatively Charged and Dark Excitons in CsPbBr3 Perovskite Nanocrystals Revealed by High Magnetic Fields. Nano Lett. 2017, 17, 6177-6183.

(27) Chen, L.; Li, B.; Zhang, C.; Huang, X.; Wang, X.; Xiao, M. Composition-Dependent Energy Splitting between Bright and Dark Excitons in Lead Halide Perovskite Nanocrystals. Nano Lett. 2018, 18, 2074 2080 .

(28) Biadala, L.; Louyer, Y.; Tamarat, P.; Lounis, B. Direct Observation of the Two Lowest Exciton ZeroPhonon Lines in Single CdSe/ZnS Nanocrystals. Phys. Rev. Lett. 2009, 103, 037404.

(29) Seong, M. J.; Mićić, O. I.; Nozik, A. J.; Cheong, A. M. M. Size-Dependent Raman Study of InP Quantum Dots. Appl. Phys. Lett. 2003, 185, 2001-2004.

(30) Brodu, A.; Ballottin, M. V.; Buhot, J.; Van Harten, E. J.; Dupont, D.; La Porta, A.; Prins, P. T.; Tessier, M. D.; Versteegh, M.; Zwiller, V.; Bals, S.; Hens, Z.; Rabouw, F. T.; Christianen, P. C.M.; De Mello 
Donega, C.; Vanmaekelbergh, D. Exciton Fine Structure and Lattice Dynamics in InP/ZnSe Core/Shell Quantum Dots. ACS Photonics 2018, 5, 3353-3362.

(31) Bayer, M.; Ortner, G.; Stern, O.; Kuther, A.; Gorbunov, A.; Forchel, A.; Hawrylak, P.; Fafard, S.; Hinzer, K.; Reinecke, T.; Walck, S.; Reithmaier, J.; Klopf, F.; Schäfer, F. Fine Structure of Neutral and Charged Excitons in Self-Assembled In(Ga)As/(Al)GaAs Quantum Dots. Phys. Rev. B 2002, 65, 195315.

(32) Javaux, C.; Mahler, B.; Dubertret, B.; Shabaev, A.; Rodina, A. V; Efros, A. L.; Yakovlev, D. R.; Liu, F.; Bayer, M.; Camps, G.; Biadala, L.; Buil, S.; Quelin, X.; Hermier, J.-P. Thermal Activation of NonRadiative Auger Recombination in Charged Colloidal Nanocrystals. Nat. Nanotechnol. 2013, 8, 206212.

(33) Langof, L.; Fradkin, L.; Ehrenfreund, E.; Lifshitz, E.; Micic, O. I.; Nozik, A. J. Colloidal InP/ZnS CoreShell Nanocrystals Studied by Linearly and Circularly Polarized Photoluminescence. Chem. Phys. 2004, 297, 93-98.

(34) De Luca, M.; Polimeni, A.; Fonseka, H. A.; Meaney, A. J.; Christianen, P. C. M.; Maan, J. C.; Paiman, S.; Tan, H. H.; Mura, F.; Jagadish, C.; Capizzi, M. Magneto-Optical Properties of Wurtzite-Phase InP Nanowires. Nano Lett. 2014, 14, 4250-4256.

(35) Liu, F.; Rodina, A. V.; Yakovlev, D. R.; Greilich, A.; Golovatenko, A. A.; Susha, A. S.; Rogach, A. L.; Kusrayev, Y. G.; Bayer, M. Exciton Spin Dynamics of Colloidal CdTe Nanocrystals in Magnetic Fields. Phys. Rev. B 2014, 89, 115306.

(36) Sercel, P. C.; Efros, A. L. Band-Edge Exciton in CdSe and Other II-VI and III-V Compound Semiconductor Nanocrystals - Revisited. Nano Lett. 2018, 18, 4061-4068.

(37) Kim, Y.-S.; Hummer, K.; Kresse, G. Accurate Band Structures and Effective Masses for InP, InAs, and InSb Using Hybrid Functionals. Phys. Rev. B 2009, 80, 035203.

(38) Rodina, A. V.; Efros, A. L. Radiative Recombination from Dark Excitons in Nanocrystals: Activation Mechanisms and Polarization Properties. Phys. Rev. B 2016, 93, 155427.

(39) Gammon, D.; Efros, A. L.; Kennedy, T. A.; Rosen, M.; Katzer, D. S.; Park, D.; Brown, S. W.; Korenev, V. L.; Merkulov, I. A. Electron and Nuclear Spin Interactions in the Optical Spectra of Single GaAs as Quantum Dots. Phys. Rev. Lett. 2001, 86, 5176-5179.

(40) Fischer, J.; Coish, W. A.; Bulaev, D. V.; Loss, D. Spin Decoherence of a Heavy Hole Coupled to Nuclear Spins in a Quantum Dot. Phys. Rev. B - Condens. Matter Mater. Phys. 2008, 78, 1-9.

(41) Biadala, L.; Shornikova, E. V.; Rodina, A. V.; Yakovlev, D. R.; Siebers, B.; Aubert, T.; Nasilowski, M.; Hens, Z.; Dubertret, B.; Efros, A. L.; Bayer, M. Magnetic Polaron on Dangling-Bond Spins in CdSe Colloidal Nanocrystals. Nat. Nanotechnol. 2017, 12, 569-574.

(42) Chekhovich, E. A.; Krysa, A. B.; Skolnick, M. S.; Tartakovskii, A. I. Direct Measurement of the HoleNuclear Spin Interaction in Single InP / GaInP Quantum Dots Using Photoluminescence Spectroscopy. Phys. Rev. Lett. 2011, 106, 027402.

(43) Kurtze, H.; Yakovlev, D. R.; Reuter, D.; Wieck, A. D.; Bayer, M. Hyperfine Interaction Mediated Exciton Spin Relaxation in (In,Ga)As Quantum Dots. Phys. Rev. B - Condens. Matter Mater. Phys. 2012, 85, 1-8.

(44) Hanson, R.; Mendoza, F. M.; Epstein, R. J.; Awschalom, D. D. Polarization and Readout of Coupled Single Spins in Diamond. Phys. Rev. Lett. 2006, 97, 1-4.

(45) Rodina, A.; Efros, A. L. Magnetic Properties of Nonmagnetic Nanostructures: Dangling Bond Magnetic Polaron in CdSe Nanocrystals. Nano Lett. 2015, 15, 4214-4222.

(46) Glazov, M. M. Electron \&amp; Nuclear Spin Dynamics in Semiconductor Nanostructures; Oxford University Press, 2018; Vol. 1, 100. 
(47) Rafipoor, M.; Dupont, D.; Tornatzky, H.; Tessier, M. D.; Maultzsch, J.; Hens, Z.; Lange, H. Strain Engineering in InP/(Zn,Cd)Se Core/Shell Quantum Dots. Chem. Mater. 2018, 30, 4393-4400.

TOC

Hyperfine interactions

Electron flip-flop

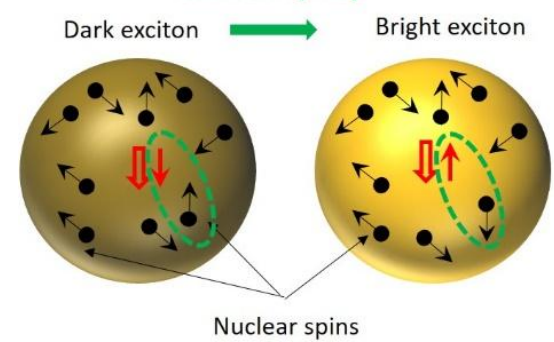

